\section{IGUALDAD DE GÉNERO Y ANÁLISIS DE LA COMUNICACIÓN MOTRIZ EN LAS TAREAS DE LA EDUCACIÓN FÍSICA}

\author{
IGUALDADE DE GÊNERO E ANÁLISE DA COMUNICAÇÃO MOTORA NAS \\ TAREFAS DE EDUCAÇÃO FÍSICA
}

\author{
GENDER EQUALITY AND ANALYSIS OF MOTOR COMMUNICATION IN \\ PHYSICAL EDUCATION TASKS
}

Javier Gil Quintana*, Joseba Etxebeste Otegi**
Palabras clave:

Educación Física. Identidad de género. Actividad física.
Resumen: El presente artículo analiza las tareas motrices propuestas en el ámbito de la Educación Física, tanto por el maestro como en el juego libre, desde una perspectiva de género. A partir de una observación participante se obtienen las tareas propuestas en las sesiones de Educación Física de una escuela en la localidad de Meliana, en la provincia de Valencia (España). Se constatan 134 tareas motrices que se analizan y contextualizan en el marco educativo. Los resultados muestran que se tiende a impulsar un cierto modelo de comportamiento común cuando el docente ejerce mayor directividad. En cambio, cuando el profesor ofrece libertad para escoger, se tienden a reproducir los estereotipos de género dominantes.
Palavras-chave: Educação Física Identidade de gênero. Atividade física.
Resumo: Este artigo analisa as tarefas motoras propostas no âmbito da Educação Física, tanto pelo professor quanto no jogo livre, a partir de uma perspectiva de gênero. A partir de uma observação participante, obtêm-se as tarefas propostas nas sessões de Educação Física de uma escola na cidade de Meliana, na província de Valência (Espanha). São analisadas e contextualizadas 134 tarefas motoras na estrutura educacional. Os resultados mostram que há uma tendência a promover um certo modelo de comportamento comum quando o professor exerce maior diretividade. Por outro lado, quando o professor oferece liberdade para escolher, há uma tendência a reproduzir os estereótipos de gênero dominantes.

Abstract: This article looks into the motor tasks proposed in Physical Education - both by teachers and in free play - from a gender perspective. Using participant observation, it collected the tasks proposed during Physical Education classes at a school in Meliana, in the Spanish province of Valencia: 134 motor tasks are analyzed and contextualized within the educational framework. The results show a tendency to promote a certain model of common behavior when teachers exercise greater directivity. On the other hand, when they offer freedom to choose, dominant gender stereotypes tend to be reproduced.
*Universidad de Valencia, Valencia, España.

E-mail: javier.gil-quintana@uv.es

**Universidad del País Vasco (UPVEHU). Vitoria-Gasteiz, España. E-mail: joseba.etxebeste@ehu.eus

Recebido em: 30-07-2018 Aprovado em: 23-03-2019 Publicado em: 24-04-2019

DOI: https://doi.org/10.22456/1982-8918.85297 (c) (i) () Licence 


\section{INTRODUCCIÓN}

Los sistemas educativos guían a los alumnos y alumnas a interiorizar los roles socioculturales de género que les permitan identificarse como hombres y mujeres capaces de vivir en sociedad con respeto y tolerancia. Es responsabilidad de la escuela promover la construcción de identidades individuales acordes con los valores del mundo de hoy: es decir, promover un equilibrio de poder y una igualdad de derechos y oportunidades entre los miembros de la sociedad con independencia de sus orientaciones sexuales y lejos de un paternalismo machista.

La educación física, como un saber más de la escuela, aborda este reto de igualdad entre mujeres y hombres siguiendo las directrices educativas aprobadas en España. La legislación pedagógica incluye entre los objetivos de la educación física la adquisición de una educación cívica que favorezca la coeducación y el respeto (COMUNIDAD VALENCIANA, 2014).

El objetivo del presente artículo es analizar las tareas motrices propuestas en el marco de las clases de educación física, tanto por el maestro como por los alumnos en el juego libre, haciendo incidencia en las diferencias que existen entre los chicos y las chicas desde una perspectiva sociopedagógica.

\section{ACTIVIDADES MOTRICES E IDENTIDAD DE GÉNERO}

La construcción de la identidad de género consiste en la integración de los atributos, normas, valores, maneras de sentir, comportamientos, hábitos, etc., considerados como femeninos y masculinos en cada contexto social. Es un proceso sociocultural que moldea una de las características derivadas de la dotación genética de cada persona como es el sexo con el que se nace (RODRÍGUEZ, 2003; SUBIRATS; TOMÉ, 2010).

La educación física contribuye en la construcción de la identidad de género mediante la participación de los niños y niñas en las actividades motrices propuestas (BORDES; COLLARD; DUGAS, 2007; GIL, 2015). Algunas autoras afirman que los juegos contribuyen a que el alumnado reproduzca comportamientos asociados a lo socialmente considerado como masculino y femenino (BONAL, 2014; THORNE, 1999; WALKERDINE, 1990). El juego supone un importante ámbito en el que comprender y practicar relaciones interpersonales y las distintas formas de ser y actuar de hombres y mujeres (LOBATO, 2007). De este modo, pueden contribuir en la construcción de la identidad de género a través de una comunicación motriz (SANTEODORO, 2015), elemento central de nuestra disertación. En estos casos, el lenguaje corporal es omnipresente y la comunicación lingüística se vuelve discreta y en muchos casos prescindible (DUGAS, 2011; PARLEBAS, 2001). En cuanto al género, esta comunicación motriz es fundamental para presentarse ante los demás como femenino o masculino (LOBATO, 2007). Se puede suponer que pudiera incluso tener más efecto en la construcción de la identidad de género que los discursos y los debates ya que estos no conllevan un cambio automático en las actitudes, en la disposición equitativa del espacio, en la voluntad de participar en juegos no considerados como propios o en la lucha para imponer otras actividades (SUBIRATS; TOMÉ, 2010). En las actividades motrices niños y niñas protagonizan roles sociomotores que están asociados a lo socialmente considerado como masculino y femenino a partir de gestos, indicios, signos y desplazamientos, es decir, a partir de comportamientos no lingüísticos (ETXEBESTE; LAVEGA; ALONSO, 2015). 
En educación física, la construcción de la identidad de género del alumnado puede verse influenciada por las interacciones sociales que tienen lugar entre los participantes. El género se crea y se recrea tanto a través de los procedimientos didácticos ejercidos por el docente cuando se comunica con el alumnado, como mediante las interacciones sociomotrices que los niños y niñas protagonizan sin mediación del docente (RONHOLT, 2002; SANTEODORO, 2015; SOLER, 2006; WRIGHT, 1995). Toda relación social contribuye a que cada sujeto busque la coherencia en la construcción de su identidad de género (FRANCIS, 2001) y la interacción motriz, como una forma más de interacción social (PARLEBAS, 2001), podría modelar la identificación de las personas como mujeres y como hombres.

La presencia 0 ausencia de interacciones motrices entre hombres y mujeres en las actividades motrices, uno de los elementos constitutivos de la lógica interna de las actividades motrices (PARLEBAS, 2001), podría tender a impulsar diferentes formas de socialización en cuanto al género. De este modo se pueden diferenciar dos tipos de relaciones en el juego. Por un lado, las tareas motrices propuestas por la escuela pueden ser sociomotrices, es decir, actividades donde la acción de un participante se ve influenciada operativamente por la acción de otro y albergar de este modo, interacciones motrices de cooperación o de oposición, que pudieran ser, a su vez, segregadoras por género o neutras, como ocurre en numerosos juegos populares (ETXEBESTE; LAVEGA; ALONSO, 2015). En ellas, niños y niñas interactúan bajo las reglas de un mismo contrato social, de un mismo contrato lúdico (PARLEBAS, 2001), de un mismo pacto con el que regular intereses compartidos, es decir, poseerían similares efectos educativos. Los juegos mixtos, de carácter neutro, pueden ejercer "una influencia positiva de unos sobre otros, una colaboración de complementariedad" (THOREL-HALLEZ, 2011, p. 83). En ellos "las conductas lúdicas se manifiestan menos estereotipadas que cuando los grupos de juego están compuestos únicamente por uno de los dos sexos" (LOBATO, 2007, p. 171), poseen una organización que limita el agrupamiento por sexos y la reproducción de una ideología del sexismo en edades de educación primaria (DEVÍS, 2001; SCRATON, 1995).

Desde el punto de vista educativo, parece que la participación no segregada por sexo del alumnado, es común en los sistemas pedagógicos basados en la educación mixta. Esta corriente pedagógica reúne a niñas y niños en espacios escolares comunes bajo el principio democrático de igualdad entre hombres y mujeres.

Por otra parte, las tareas que se proponen en educación física pueden estar exentas de interacciones motrices entre chicos y chicas. Así ocurre en las tareas denominadas psicomotrices o individuales, como en un lanzamiento de jabalina en atletismo, donde no existe interacción motriz entre los participantes.

Las tareas motrices diferenciadas tienden a estar presentes en los sistemas educativos basados en la educación diferenciada, corriente pedagógica en la que los niños aprenden en unos espacios escolares y las niñas en otros. Para esta perspectiva los chicos poseen de forma natural más ventajas cognitivas a la hora de desarrollar capacidades como la representación espacial o la competencia matemática y tienden a ser más agresivos, dominantes y físicamente activos. Las chicas son mejores en el campo de las destrezas lingüísticas o las expresivas, suelen ser más cooperativas, disciplinadas y muestran más interés (HOFF, 2005; MARTIAL, 2005; MEVES, 2005). Desde este punto de vista estas diferencias hacen necesaria la separación del alumnado para evitar el surgimiento de desigualdades que provienen de manera innata, algo recurrente en la educación mixta en la que según la educación diferenciada los estereotipos 
se radicalizan, el rendimiento escolar es más bajo, la violencia de género es más elevada y el conocimiento del sexo opuesto se desvirtúa ya que niños y niñas se muestran tal y como al otro sexo le gustaría que fuese (CALVO, 2005).

Sin embargo, desde otros puntos de vista, las actividades diferenciadas no favorecen la igualdad sino todo lo contrario. Unas prácticas motrices como son las deportivas, dotadas de competición, aupadas por las instituciones y tradicionalmente segregadas, han sido espacios de socialización masculina pero no femenina debido a la consideración del deporte como ámbito masculino (ETXEBESTE; LAVEGA; ALONSO, 2015). Según Díez (2006) las prácticas deportivas "han constituido una forma de socialización específica que ha iniciado a muchos jóvenes varones en marcos de referencia amplios e importantes y en habilidades que les preparan para situarse en la estructura social" (p. 136) mientras que han dejado fuera a las féminas para adquirir aprendizajes y ocupar los centros simbólicos de poder y prestigio. Si bien en el deporte hay cada vez más una mayor participación de las mujeres, una modificación en la percepción de aquello que es considerado como masculino y femenino, un cambio en los valores occidentales y nuevas formas para modelarse como persona fuera de los estereotipos de género (MOSQUERA; PUIG, 2009), el deporte sigue siendo una esfera en la que el rol masculino es el imperante (GRIFFIN, 1985; MESSNER, 2007). Es un ámbito inigualable de la vida cultural según Hargreaves (1993) "en el que se fomenta el sexismo y en donde las mujeres están seriamente discriminadas" ( $p$. 109). El deporte, como laboratorio excepcional de comportamiento social (PARLEBAS, 2001) es realmente según Klein (1990) un laboratorio de masculinidad.

Asimismo, desde el punto de vista pedagógico y según las perspectivas basadas en la coeducación, es decir, en la formación del alumnado en la igualdad y en el cuestionamiento de los roles de género tradicionales, las actividades diferenciadas limitan a la hora orientar al alumnado a que aprenda el significado de la igualdad. Si bien son prácticas que podrían ser beneficiosas al ser utilizadas de manera puntual (SUBIRATS; TOMÉ, 2010), en ellas chicos y chicas pueden tener dificultades para comprender el sentido de una convivencia social conjunta y más aún en condiciones equitativas de derechos y oportunidades. Desde el prisma de la coeducación se defiende que la educación diferenciada trata de hacer hincapié exclusivamente en el rendimiento académico y a hacerlo además desde posiciones puramente ideológicas y con poco rigor científico. Según Halpern et al. (2011) los argumentos de la educación diferenciada no son sólidos, las diferencias cerebrales entre chicas y chicos no justifican una educación dual y lo único que se consigue con ella es consolidar los estereotipos sexistas.

Conociendo la diversa influencia que las actividades motrices pueden ejercer en la construcción de la identidad de género, este estudio analiza cómo la educación física de un contexto concreto modela la identificación del alumnado como los hombres y las mujeres de la sociedad del hoy y del mañana.

\section{METODOLOGÍA}

\subsection{PROCEDIMIENTO}

Para observar cómo la educación física promueve la identificación del alumnado como hombres y mujeres a través de las actividades motrices, cómo construye su identidad de género 
mediante hechos y no palabras, mediante la práctica y no la teoría, esta investigación se apoya en la etnografía. Es un método tradicionalmente empleado en educación para desentrañar aspectos que a menudo permanecen latentes, para mostrar "capas de significación que permanecen ocultas a la observación superficial y que a menudo son diferentes de lo que se supone que son" (WOODS, 1987, p. 21). En educación física permite ver un lenguaje corporal intencional y convencional que sustituye al lingüístico (DUGAS, 2011) como hace por ejemplo Santeodoro (2015) en cuanto a la reproducción de los modelos de género.

Desde noviembre de 2008 hasta julio de 2009 y tras obtener los permisos pertinentes se observan 80 sesiones de educación física del colegio público Mediterrani de Meliana, una localidad de la provincia de Valencia (España). El número de sesiones supone el $20 \%$ aproximadamente del total del curso escolar. A las sesiones acuden 134 niños y niñas de entre 6 y 12 años repartidos en los seis cursos de la etapa de educación primaria. Para la observación se permanece en un lateral del gimnasio o de los patios para no entorpecer el curso de las clases y para preservar la intimidad de los niños y niñas, un público condicionado como dice Rodríguez (2007) por un sistema de protección asociado a un distinto eje generacional.

Durante la observación se anotan en un cuaderno de campo las tareas motrices realizadas por los niños y niñas para poner de manifiesto el tipo de educación que promueven los contenidos de la educación física como opciones culturales mediante las que construir los significados de la enseñanza (CONTRERAS, 1998). Se anota cada actividad como si fuera una acción nueva para evitar que los investigadores den por hecho las características y las consecuencias de la realización (OIARBIDE, 2009).

A partir de las anotaciones se elabora una ficha por cada tarea, algo común en la tradición praxiológica desde hace más de 3 décadas (ETXEBESTE, 2001; GIL, 2017; OULD SALEK, 1994; PARLEBAS, 1987; SANTEODORO, 2015). La utilización de las fichas permite como dice Vigne (2006) componer un repertorio cuasi-exhaustivo de actividades para dar coherencia a los datos e interpretar con mayor rigor las actividades motrices observadas. El número de fichas obtenidas es de 134. En cada ficha se anotan aspectos generales como la descripción de la actividad o la fecha de realización. Se incluyen también los aspectos de la lógica externa o las características sociales e individuales de las prácticas como es el género o la edad de los participantes. Por último, se añaden los aspectos de la lógica interna (Parlebas, 2001), es decir los rasgos pertinentes de las situaciones motrices como son los tipos de interacción motriz.

\subsection{ANÁLISIS}

Para el análisis de las fichas se opta por un examen cuantitativo y otro cualitativo debido a las particularidades de la observación realizada y a las características de los datos recogidos. Esto es así ya que se constata un fenómeno mayor: la existencia, por un lado, de tareas dirigidas por el profesor en el que el docente ordena los grupos de un modo mixto y en el que todos los discentes ejecutan el mismo quehacer y, por otro, de "tiempo libre", llamado por el profesor "deporte libre", en el que el alumnado se organiza en función de su voluntad, tanto en los grupos, que son segregados entre chicos y chicas, como la tarea motriz en la que se sumergen. De este modo, en el caso de las tareas dirigidas se observó y anotó la duración de cada una, mientras que en las fases de "deporte libre" resultó imposible con las 
herramientas que se disponían, por lo que muestran, de un modo más global, las tendencias de la comunicación motriz.

De manera cuantitativa se analizan las tareas dirigidas. Son 82 actividades realizadas por todo el alumnado tras una descripción detallada de las normas por parte del docente: al mismo tiempo todo el alumnado corre por la pista de atletismo, o se pasa la pelota por parejas en la cancha, o juega a béisbol en todo el patio. Desde el punto de vista metodológico estas actividades permiten un análisis cuantitativo para comprobar la tendencia con la que se distribuyen los valores de una misma variable en función del tiempo total de realización. A partir de las categorías expuestas en las fichas se elabora una base de datos con el programa estadístico SPSS versión 15.0 con el que se realiza un análisis de comparación de medias. La variable independiente es el género y la variable dependiente el tiempo total de realización de las actividades (24 horas, 11 minutos y 30 segundos). Se elaboran gráficos descriptivos con el programa Microsoft Excel para representar los porcentajes de la suma del tiempo de cada variable. Desde el punto de vista educativo el análisis de estas tareas permite además observar qué construcción de la identidad de género impulsa el docente para todo el alumnado ya que son actividades que tienden a promover un proceso de socialización homogéneo y un menor desarrollo de la autonomía (DÍAZ, 1993; JOVER, 1999).

De manera cualitativa se analizan las actividades auto-gestionadas. Son 52 situaciones motrices elegidas por el alumnado durante momentos de juego libre concedidos por el docente. Desde el punto de vista metodológico son actividades que requieren un análisis descriptivo para mostrar la predisposición del alumnado para interactuar en función del género. Distintos juegos se solapan en un mismo espacio y momento temporal: mientras un grupo de niños juega un partido de fútbol, un alumno corre a solas por el patio, una pareja se pasa la pelota y una niña lanza la pelota de baloncesto a canasta. Los resultados se muestran en tablas de tendencias que reflejan la tendencia con la que el alumnado juega de manera mixta 0 diferenciada. Desde el punto de vista educativo el análisis de las actividades auto-gestionadas permite además constatar qué identidad de género construye cada alumno y alumna a partir de sus preferencias y estereotipos ya que el juego libre da libertad a los niños y niñas para decidir por sí mismos con autonomía e iniciativa (BLÁNDEZ, 2005; JOVER, 1999; LORENTE, 2008).

Los datos de la comunicación motriz de ambos análisis, el cuantitativo y el cualitativo, se analizan a partir de la praxiología motriz para conocer las consecuencias específicas de la acción motriz, de aquella "que cobra sentido y se concreta en la intervención del cuerpo, en la actualización de las conductas motrices" (PARLEBAS, 2001, p. 356), lo que permite comprender los efectos de las actividades motrices en la educación física, entendida como una práctica de intervención pedagógica cuyo objetivo es modelar las conductas motrices del alumnado. Asimismo, los datos se interpretan a partir de las ciencias de la educación para dar cuenta de las particularidades pedagógicas de la práctica educativa, para poner de manifiesto la óptica del profesorado a la hora de organizar la realidad (CHÁVEZ; DELER; SUÁREZ, 2008). Interpretar los datos a partir de las ciencias de la educación permite dilucidar los efectos pedagógicos de la realización de las actividades motrices propuestas por el profesorado de educación física en cuanto al género. 


\section{RESULTADOS Y DISCUSIÓN}

\subsection{EL JUEGO DIRIGIDO: UNA IGUALDAD A MEDIO CAMINO}

En análisis de la organización de los grupos en las actividades dirigidas por el docente, es decir, cuando el profesor propone las tareas y organiza los chicos y las chicas, muestra que el alumnado realiza durante un $48,6 \%$ (705 minutos) situaciones motrices mixtas y en un $20,5 \%$ (298 minutos) situaciones motrices diferenciadas. Asimismo, en un 30,9\% (448,5 minutos) el alumnado realiza prácticas psicomotrices, actúa de manera individual, no se relaciona ni de manera mixta ni diferenciada.

Figura 1 - Actividades dirigidas mixtas, diferencias y sin interacción motriz. Se observa una mayor tendencia a la realización de actividades mixtas.

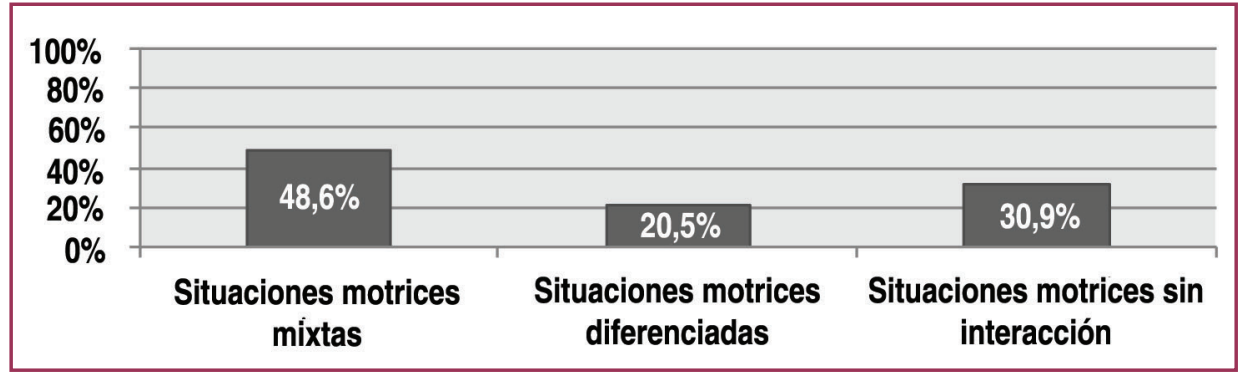

Fuente: datos del análisis cuantitativo

El análisis muestra que en las actividades dirigidas los niños y niñas interactúan en casi 5 de cada 10 ocasiones. Como ocurre por ejemplo en los partidos de béisbol, baloncesto o pelota valenciana, chicas y chicos corren en los mismos espacios, comparten objetos, se relacionan a través de signos e indicios, persiguen objetivos, mantienen contacto cuerpo a cuerpo, juegan uno contra todos o todos contra uno, comparten un mismo contrato lúdico independientemente de su sexo biológico. De esta manera, el alumnado realiza actividades mixtas que, si bien no es una solución inmediata para favorecer la coeducación, sí que reducen los estereotipos y aumentan la complementariedad (DEVÍS, 2001; LOBATO, 2007; THORELHALLEZ, 2011) y suponen un cambio respecto a la escolarización segregada tradicional a la hora de promover la igualdad de género (SUBIRATS; TOMÉ, 2010).

Si consideramos a su vez, la comunicación motriz propuesta por las tareas, y la organización de los grupos mixtos o segregados se constata que en 2 de cada 10 ocasiones el alumnado participa en tareas diferenciadas, los niños con los niños y las niñas con las niñas, y en 3 de cada 10 actúa de forma individual en las tareas psicomotrices, cada alumno corre por su cuenta en el calentamiento y cada alumna realiza los estiramientos sin interactuar con sus compañeros o compañeras. Así, los datos muestran que en la mitad de la intervención el alumnado, a pesar de compartir un mismo espacio educativo, no interactúa entre niñas y niños, no emite ni lee mensajes de personas de diferente género, los chicos no mantienen contacto directo con las chicas, las niñas no pasan la pelota a los niños, no deciden qué agrupaciones formar ni qué metas perseguir conjuntamente.

El examen refleja de esta manera, unas tareas dirigidas que están a medio camino a la hora de impulsar la igualdad. La intervención no se caracteriza por facilitar mayoritariamente la 
relación social entre hombres y mujeres, por fomentar de manera intensa una intersubjetividad común, por promover proyectos compartidos, por favorecer agrupaciones de niñas y niños mediante los que caminar hacia la coeducación (GIL, 2015; RODRÍGUEZ, 2003; THORNE, 1999). El profesor promueve un proceso de socialización homogéneo caracterizado por un equilibrio entre proyectos compartidos y proyectos sin relación social entre niñas y niños, entre una socioafectividad igual para ellos y ellas y otra en la que la diferencia sexual es la imperante.

\subsection{EL JUEGO LIBRE: REPRODUCTOR DE LOS ESTEREOTIPOS DE GÉNERO}

El análisis de las situaciones motrices auto-gestionadas muestra que tienden a ser mayoritariamente diferenciadas. Cuando el profesor encomienda llevar a cabo el juego libre los chicos juegan con los chicos y las chicas con las chicas. Algunos discentes, de un modo aislado, realiza actividades psicomotrices, atraído principalmente por el uso libre del material y sin establecer relaciones con otros, niñas o niños, aunque poco a poco son absorbidos por las actividades mayoritarias de su grupo.

Cuadro 1 - Tareas auto-gestionadas mixtas, diferenciadas y sin interacción motriz. Se observa una tendencia mayoritaria hacia la realización de las segundas.

\begin{tabular}{cccc}
\hline & Mixtas & Diferenciadas & Sin interacción \\
\hline $\begin{array}{c}\text { Actividades auto- } \\
\text { gestionadas }\end{array}$ & Muy minoritarias & Muy mayoritarias & Minoritarias \\
\hline
\end{tabular}

Fuente: datos del análisis cualitativo

En el juego libre de la escuela los niños aprenden a relacionarse con los niños y las niñas con las niñas, ellos a emocionarse con ellos y ellas con ellas, los chicos a desarrollar una socioafectividad particular y las chicas otra.

Además, si tenemos en cuenta la lógica interna de las tareas auto-gestionadas, el sistema de reglas acordado, vemos que impulsa en términos generales actividades de relación social, es decir, actividades sociomotrices. No obstante la naturaleza de la comunicación motriz es diferente. Los chicos tienden a elegir actividades con interacciones motrices de cooperaciónoposición como un partido de fútbol y de oposición como los duelos de uno contra uno. Las chicas en cambio tienden a protagonizar actividades de cooperación motriz como los saltos a la comba.

Cuadro 2 - Lógica interna de las tareas auto-gestionadas protagonizadas por chicos o por chicas. Se observa una tendencia de los chicos para realizar actividades de oposición y de cooperación-oposición y de las chicas para llevar a cabo actividades cooperativas.

\begin{tabular}{lcccc}
\hline & Psicomotricidad & Cooperación & Oposición & $\begin{array}{c}\text { Cooperación- } \\
\text { Oposición }\end{array}$ \\
\hline Actividades de chicos & Minoritarias & Minoritarias & Mayoritarias & Mayoritarias \\
Actividades de chicas & Minoritarias & Mayoritarias & Minoritarias & Minoritarias \\
\hline
\end{tabular}

Fuente: datos del análisis cualitativo

En el juego libre concedido por el docente los chicos siguen unos tipos de relación motriz y las chicas otro. Como ocurre en otros ámbitos, en la escuela Mediterrani de Meliana 
los niños aprenden a enfrentarse y a competir con niños y las niñas a cooperar y a ser solidarias con niñas (DÍEZ, 2006; GRIFFIN, 1985; MESSNER, 2007; SANTEODORO, 2015) hecho que dilucida el poder de la lógica interna para orientar la afectividad en cuanto al género (BORDES; COLLARD; DUGAS, 2007; ETXEBESTE; LAVEGA; ALONSO, 2015). La comunicación motriz de las tareas escogidas impulsa diferentes formas de decisión, emoción y relación social.

El análisis de las actividades auto-gestionadas refleja unas tareas mediante las que construir una identidad de género basada en la diferencia. Los chicos juegan partidos de fútbol y las chicas a la comba, ellos ocupan unos espacios como el campo de fútbol sala y ellas los porches o las escaleras, los niños utilizan unos objetos y las niñas otros, ellos aprenden a emocionarse con ellos y ellas a establecer interacciones personales con sus compañeras. El docente promueve la igualdad en la libertad de escoger sus propias tareas pero al mismo tiempo permite que el propio alumnado pueda reproducir los procesos de socialización diferenciados bajo el estandarte de la libertad. Como tiende a ocurrir en los ámbitos pedagógicos de menor regulación, la libertad contribuye a reproducir desigualdades antes que a subsanarlas (SOLER, 2006), la espontaneidad de conductas y actitudes tiende a facilitar un tipo de interacción que refuerza las diferencias entre lo masculino y lo femenino (BONAL, 1997). La no intervención, el liberalismo que caracteriza el juego libre, da libertad para elegir a partir de los intereses individuales, pero no para caminar hacia una convivencia social basada en relaciones igualitarias entre hombres y mujeres.

\section{CONCLUSIONES}

La educación física de Meliana bascula al alumnado de un lado a otro a la hora de orientarle a construir su identidad de género. Por un lado, cuando el profesor tiene mayor control de las actividades, tiende a promover en mayor medida proyectos mixtos en los que aprender a compartir entre niñas y niños. Por otro lado, cuando el docente deja al alumnado que tenga iniciativa para protagonizar sus propios juegos, chicos y chicas se separan en tareas individuales y diferenciadas en las que se segrega y en las que reproduce los estereotipos de género dominantes. De esta manera, de manera consciente o inconsciente, la educación física parece impulsar un mundo de relación social en el que las diferencias entre ser niño o ser niña están desdibujadas y al mismo tiempo permite reproducir una sociedad cuya ciudadanía se separa bajo criterios de segregación sexual.

Con el objetivo de promover una enseñanza más coeducativa, sería recomendable que la educación física reflexionara sobre la libertad de elección de las tareas del alumnado, que tienden a reproducir los sistemas imperantes de género y buscara la promoción de actividades sin sesgo de género. Se hacen necesarias actividades diferentes (DELCASTILLO-ANDRÉS; CAMPOS-MESA; CASTAÑEDA; GONZÁLEZ, 2016), en las que las niñas estén motivadas, en espacios no identificados socialmente como masculinos o femeninos, basadas sobre todo en la cooperación, que estén lideradas tanto por niñas como por niños y en las que se establezcan estrategias para evitar una participación pasiva (LASAGA; RODRÍGUEZ, 2006; MORENO, 2013). Hacerlo puede contribuir a construir una sociedad en la que niñas y niños sepan convivir con mismos derechos y deberes y no supeditados a relaciones de poder entre unos y otras. 


\section{REFERENCIAS}

BLÁNDEZ, Julia. La utilización del material y del espacio en educación física: Propuestas y recursos didácticos. Barcelona: Inde, 2005.

BONAL, Xabier. Las actitudes del profesorado ante la coeducación: Propuestas de intervención. Barcelona: Grao, 1997.

BONAL, Xabier. Cambiar la escuela: la coeducación en el patio de juegos. Barcelona: UAB, 2014. (Cuadernos para la Coeducación, D1).

BORDES, Pascal; COLLARD, Luc; DUGAS, Eric. Vers une science des activités physiques et sportives: La science de l'action motrice. París: Vuibert, 2007.

CALVO, María. Todos iguales pero diferentes. El derecho a una educación diferenciada. In: BARRIO, José María (ed.). Educación diferenciada, una opción razonable. Barañain: Universidad de Navarra, 2005. p. 263-298.

CHÁVEZ, Justo; DELER, Gustavo; SUÁREZ, Amparo. Principales corrientes y tendencias a inicios del siglo XXI de la Pedagogía y la Didáctica. La Habana: Instituto Central de Ciencias Pedagógicas, 2008.

COMUNIDAD VALENCIANA. Decreto 108/2014, de 4 de julio, por el que se establece el currículo y desarrolla la ordenación general de la educación primaria en la Comunitat Valenciana. Diario Oficial de la Comunitat Valenciana, Valencia, n. 7311.

CONTRERAS, Onofre Ricardo. Didáctica de la educación física: Un enfoque constructivista. Barcelona: Inde, 1998.

DELCASTILLO-ANDRÉS, Óscar; CAMPOS-MESA, María del Carmen; CASTAÑEDA, Carolina; GONZÁLEZ, Gloria; Estudio observacional del sexismo en la educación física del sur de España. Movimento, v. 22, p. 263-278, 2016.

DEVÍS, José. El currículum oculto y las nuevas orientaciones en el estudio del currículum en la Educación Física. In: VÁZQUEZ, Benilde (coord.). Bases educativas de la actividad física y el deporte. Madrid: Síntesis, 2001, p. 278-299.

DÍAZ, Arturo. El juego como actividad de enseñanza y/o aprendizaje: adaptaciones. In: DÍAZ, Arturo; GARCÍA, Juan José; CONTE, María Luisa; RIVADENEYRA, Juan; MALLO, Pedro; ÁLVAREZ, Jesua. Desarrollo curricular para la formación. Madrid: Gymnos, 1993, p. 329-348.

DÍEZ, Carmen. Juventud y deporte. Una propuesta de cambio en la organización tradicional de la actividad deportiva. Revista de Dialectología y Tradiciones Populares, v. 66, n. 2, p. 129144, jul./dic. 2006.

DUGAS, Eric. L'homme systémique: Pour comprendre les pratiquants des jeux sportifs. Nancy: Presses Universitaires de Nancy, 2011.

ETXEBESTE, Joseba. Les jeux sportifs, eléments de la socialisation traditionelle des enfants du Pays Basque. 2001. 188p. Tesis (doctorado) - Université Paris V-René Descartes, París, 2001.

ETXEBESTE, Joseba; LAVEGA, Pere; ALONSO, Ignacio. Dominios de acción motriz, género y emociones: una investigación sobre los efectos de la lógica interna de los juegos deportivos 
en las emociones de hombres y mujeres. In: CONGRESO INTERNACIONAL PARA EL IMPULSO DE POLÍTICAS DE IGUALDAD DE MUJERES Y HOMBRES, 2012. [Anais...] Bilbao: Emakunde, Instituto Vasco de la Mujer, 2015.v.3, p. 347-358.

FRANCIS, Becky. Beyond postmodernim: feminist agency in educational research. In: FRANCIS, Becky; SKELETON, Christine (eds.). Investigating gender: Contemporary perspectives in education. Buckingham: Open University 2001. p. 65-76.

GIL, Javier. Las dificultades del deporte para fomentar la coeducación: el caso del colpbol. In: CONGRESO INTERNACIONAL PARA EL IMPULSO DE POLÍTICAS DE IGUALDAD DE MUJERES Y HOMBRES, 2012. Bilb: Emakunde, Instituto Vasco de la Mujer, 2015. v. 3, p. 387398.

GIL, Javier. La socializacion oculta en educación física. Análisis de la enseñanza de las actividades físicas y deportivas en Meliana (València). 2017. 394p. Tesis (doctorado) Departamento de Educación Física y Deporte, Universidad del País Vasco, Vitoria-Gasteiz, 2017.

GRIFFIN, Patricia S. "Boys" Participation Styles in a Middle School Physical Education Team Sports Unit. Journal of Teaching in Physical Education, v. 4, p. 100-110, ene./mar. 1985.

HALPERN, Diane F.; ELIOT, Lise; BIGLER, Rebecca S.; FABES, Richard A.;HANISH, Laura D.; HYDE, Janet; LIBEN, Lynn S.; MARTIN, Carol Lynn. The Pseudoscience of Single-Sex Schooling. Science, v. 333, p. 1706-1707, sep. 2011.

HARGREAVES, Jennifer. Promesa y problemas en el ocio y los deportes femeninos. In: BARBERO, José Ignacio (ed.). Materiales de sociología del deporte. Madrid: La Piqueta, 1993. p. 109-132.

HOFF, Christina. Educación diferenciada: un activo para una sociedad plural. In: BARRIO, José Maria. Educación diferenciada, una opción razonable. Barañain: EUNSA, 2005. p. 237-248.

JOVER, Roberto. Juegos motores. Fundamentos para el diseño y presentación de juegos didácticos motrices en educación primaria. In: VILLAMÓN, Miguel. Formación de los maestros especialistas en Educación Física. Valencia: Conselleria de Cultura, Educació i Ciència. Generalitat Valenciana, 1999. p. 156-167.

KLEIN, Michael. The macho world of sport - a forgotten realm? Some introductory remarks. International review for the Sociology of Sport, v. 25, n. 3, p. 175-184, sep. 1990.

LASAGA, María José; RODRÍGUEZ, Carmen. La coeducación en la educación física y el deporte escolar: liberar modelos. Sevilla: Wanceulen, 2006.

LOBATO, Emma. Juego y género. Estudio etnográfico sobre la construcción de la identidad de género en los contextos y prácticas lúdicas infantiles. 2007. 506p. Tesis (doctorado) - Departamento de Ciencias de la Educación, Universidad de Oviedo, Oviedo, 2007.

LORENTE, Eloísa. Estimular la responsabilidad y la iniciativa: autogestión en educación física. Apunts: Educació física i esports, n. 92, p. 26-34, 2008.

MARTIAL, Ingbert von. Coeducación y educación separada. In: BARRIO, José Maria. Educación diferenciada, una opción razonable. Barañain: EUNSA, 2005. p. 21-88.

MESSNER, Michael A. Out of Play. Albany: Suny, 2007. 
MEVES, Christa. Las chicas son diferentes y los chicos más. In: BARRIO, José Maria.

Educación diferenciada, una opción razonable. Barañain: EUNSA, 2005. p. 249-262.

MORENO, María Antonia. Queremos coeducar. Avilés: Centro de Profesorado y Recursos de Avilés-Occidente, 2013.

MOSQUERA, María José; PUIG, Nuria. Género y edad en el deporte. In: GARCÍA, Manuel; PUIG, Nuria; LAGARDERA, Francisco (comps). Sociología del deporte. Madrid: La Piqueta, 2009. p. 109-132.

OIARBIDE, Asier. Mendi zeharkaldi, aerobic eta futbolaren etnomotrizitateak. 2009. 483p. Tesis (doctorado) - Departamento de Educación Física y Deporte, Universidad del País Vasco, Vitoria-Gasteiz, 2009.

OULD SALEK, Mohamed. Les Jeux Sportifs de L'Afrique de l'ouest pre-coloniale : une étnomotricité originale. 1994. 564f. Tesis (doctorado) - Université Paris V - René Descartes, París, 1994.

PARLEBAS, Pierre. Fiche d'observation d'un jeu. Vers l'éducation nouvelle. Paris : Hors serie, 1987. p. 192-212.

PARLEBAS, Pierre. Juegos, deporte y sociedades:Léxico de praxiología motriz. Badalona: Paidotribo, 2001.

RODRÍGUEZ, Ivan. Para una sociología de la infancia: aspectos teóricos y metodológicos. Madrid: CIS, 2007.

RODRÍGUEZ, María del Carmen. La configuración del género en los procesos de socialización. Oviedo: KRK, 2003.

RONHOLT, Helle. 'It's only the sissies...': analysis of teaching and learning processes in physical education: a contribution to the hidden curriculum. Sport, Education and Society, n. 6, p. 2536, 2002.

SANTEODORO, Yolanda. Kurutziaga Ikastolako gorputz hezkuntzan giza genero eredu motorrak. 2015. 256p. Tesis (doctorado) - Departamento de Educación y Deporte, Universidad del País Vasco, Vitoria-Gasteiz, 2015.

SCRATON, Sheila. Educación física de las niñas: un enfoque feminista. Madrid: Morata, 1995.

SOLER, Susana. Actitudes y relaciones de niñas y niños ante contenidos de la educación física de primaria estereotipados por el género: el caso del fútbol. In: VÁZQUEZ, Benilde (coord.). Las mujeres jóvenes y las actividades físico-deportivas. Madrid: Consejo Superior de Deportes, 2006.p. 116-146.

SUBIRATS, Marina; TOMÉ, Amparo. Balones fuera. Reconstruir los espacios desde la coeducación. Barcelona: Octaedro, 2010.

THORNE, Barrie. Gender play: Girls and Boys in School. Rutgers, NJ: Rutgers University Press, 1999.

THOREL-HALLEZ, Sabine. De la mixité à la coéducation en danse contemporaine au collège : Analyse de l'activité d'enseignant-e-s d'éducation physique et sportive. París: Harmattan, 2011. 
VIGNE, Mickaël. Étude sociologique intraculturelle des jeux traditionnels dans l'espace linguistique picard. 2006. 686p. Tesis (doctorado) - Université René Descartes - Sorbonne, París, 2006.

WALKERDINE, Valerie. Schoolgirl Fictions. Londres: Verso, 1990.

WOODS, Peter. La escuela por dentro: La etnografía en la investigación educativa. Barcelona: Paidós y Ministerio de Educación y Ciencia, 1987.

WRIGHT, Jan. Feminist poststructuralist methodology for the study of gender construction in physical education: description of a study. Journal of teaching in physical education, v. 15, p. 1-24, 1995. 